\title{
CONTRIBUCIÓN AL ESTUDIO POLÍNICO DE ESPECIES ORNAMENTALES: BIGNONIACEAE
}

\author{
$M^{\mathrm{a}}$ del Mar TRIGO
}

\begin{abstract}
RESUMEN. Se estudian con el microscopio óptico (MO) y con el microscopio electrónico de barrido (MEB) la morfología polínica de 12 especies de Bignoniaceae habitualmente cultivadas como ornamentales en la Región Mediterránea.

Los táxones estudiados se pueden dividir en 5 clases en base a la morfología polínica que presentan: Campsis grandiflora, C. radicans y Spathodea campanulata poseen granos de polen trizonocolporados; Doxantha unguis-cati, Pandorea jasminoides, P. pandorana, Podranea ricasoliana, y Tecomaria capensis presentan granos de polen trizonocolporoidados; en Jacaranda acutifolia el polen es tricolpado y en Pyrostegia ignea 12-pantocolpado mientras que Catalpa bignonioides y Tecoma stans tienen los granos de polen agrupados en tétrades. No obstante, los géneros incluidos en una misma clase se han diferenciado, por tipos, en base a la ornamentación que presenta la superficie del grano de polen.
\end{abstract}

Palabras clave. Polen, ornamentales, Bignoniaceae, España.

SUMMARY. The pollen morphology of twelve species of the genera Campsis, Catalpa, Doxantha, Jacaranda, Pandorea, Podranea, Pyrostegia, Spathodea, Tecoma and Tecomaria, usually cultivated in the Mediterranean Region are studied by light and scanning electron microscopy. Pollen was acetolysed by the method of Erdtman (1960) modificated by Reitsma (1969) and mounted in glycerine jelly for light microscopy. For scanning electron microscopy the pollen were coated of gold evaporate.

Centered in the tropics, particulary South America, this family is primarily entomophilous and their species has been shown as melliferous, but the numerous showy flowers of some species may shed pollen and to produce local concentracition in atmosphere and be caught by spore-trap.

The twelve taxa examined can be divided into five well defined types: Campsis grandiflora, C. radicans and Spathodea campanulata have 3-colpate pollen; Doxantha unguiscati, Pandorea jasminoides, P. pandorana, Podranea ricasoliana, and Tecomaria capensis have 3-colporoidate pollen; Jacaranda acutifolia have 3-colpate pollen; Pyrostegia ignea have 12-colpate pollen while Catalpa bignonioides and Tecoma stans have pollen grains agrupate in tetrades. However, the genera included in a same type can be separate on the basis of the sexine patterns.

Key words. Pollen, ornamentals, Bignoniaceae, Spain.

\section{INTRODUCCIÓN}

La familia Bignoniaceae agrupa alrededor de 120 géneros y unas 650 especies con distribución centrada en las regiones tropicales, fundamentalmente de Sudamérica. 
Debido a que poseen flores grandes y vistosas, se ha difundido mucho su cultivo como plantas ornamentales, especialmente en zonas de clima cálido y subtropical, en donde se ha podido observar que sus flores son visitadas repetidamente por las abejas atraidas por el abundante néctar que segregan y por la viva coloración de la corola. Por esta razón resulta bastante probable la aparición del polen de estas plantas en el contenido de mieles procedentes de colmenas próximas a núcleos urbanos.

Por otra parte, algunas de estas especies son árboles de gran tamaño, con una elevada producción de flores, que fácilmente pueden provocar concentraciones, más o menos locales, de polen en la atmósfera y ser atrapados por los captadores utilizados en aerobiología. De hecho, el polen de algunas de las especies aquí estudiadas ha sido citado como aerovagante por Suárez y Seoane (1983) y Swineford (1940) sec. Lewis et al. (1983).

El presente trabajo pretende ser una continuación de otros ya publicados anteriormente (Trigo, 1989; Trigo y García, 1990), con objeto de completar el estudio polínico de especies ornamentales.

\section{MATERIAL Y MÉTODOS}

Para el estudio polínico se ha utilizado tanto material fresco, recién recolectado e incluido en ácido acético glacial, como material seco procedente del Herbario del Departamento de Biología Vegetal de la Facultad de Ciencias de la Universidad de Málaga (MGC). En todos los casos dicho material se trató siguiendo el método acetolítico de Erdtman (1960) modificado por Reitsma (1969). El montaje para su observación con el microscopio óptico se realizó usándose como medio glicerogelatina incolora y sellándose posteriormente las preparaciones con parafina. Para su observación con el microscopio electrónico de barrido (MEB) se recubrieron las muestras con oro vaporizado en atmósfera de alto vacío, apareciendo en cada una de las microfotografías obtenidas las siguientes inscripciones, de izquierda a derecha: número de archivo de la foto, kilovoltaje al que fue sometida la muestra, microescala y distancia de trabajo (WD), expresada en milímetros.

Para calcular las dimensiones de los ejes polar (P), ecuatorial (E) y diámetro (D), se han efectuado 30 medidas por muestra y para los demás caracteres al menos 15 observaciones. Los valores que aparecen entre paréntesis corresponden a la media aritmética y a la desviación típica del intervalo.

La terminología empleada en las descripciones es, fundamentalmente, la propuesta por Erdtman (1945, 1952), Faegri \& Iversen (1975), Hideux \& Ferguson (1975), Reitsma (1970), Van Campo (1958) y Wodehouse (1935).

En el texto, las especies aparecen ordenadas alfabéticamente, habiendose agrupado aquéllas cuyos granos de polen presentan una morfología similar. Se incluye, además, el areal natural de distribución de las mismas así como las referencias que hemos encontrados sobre estudios anteriores. 

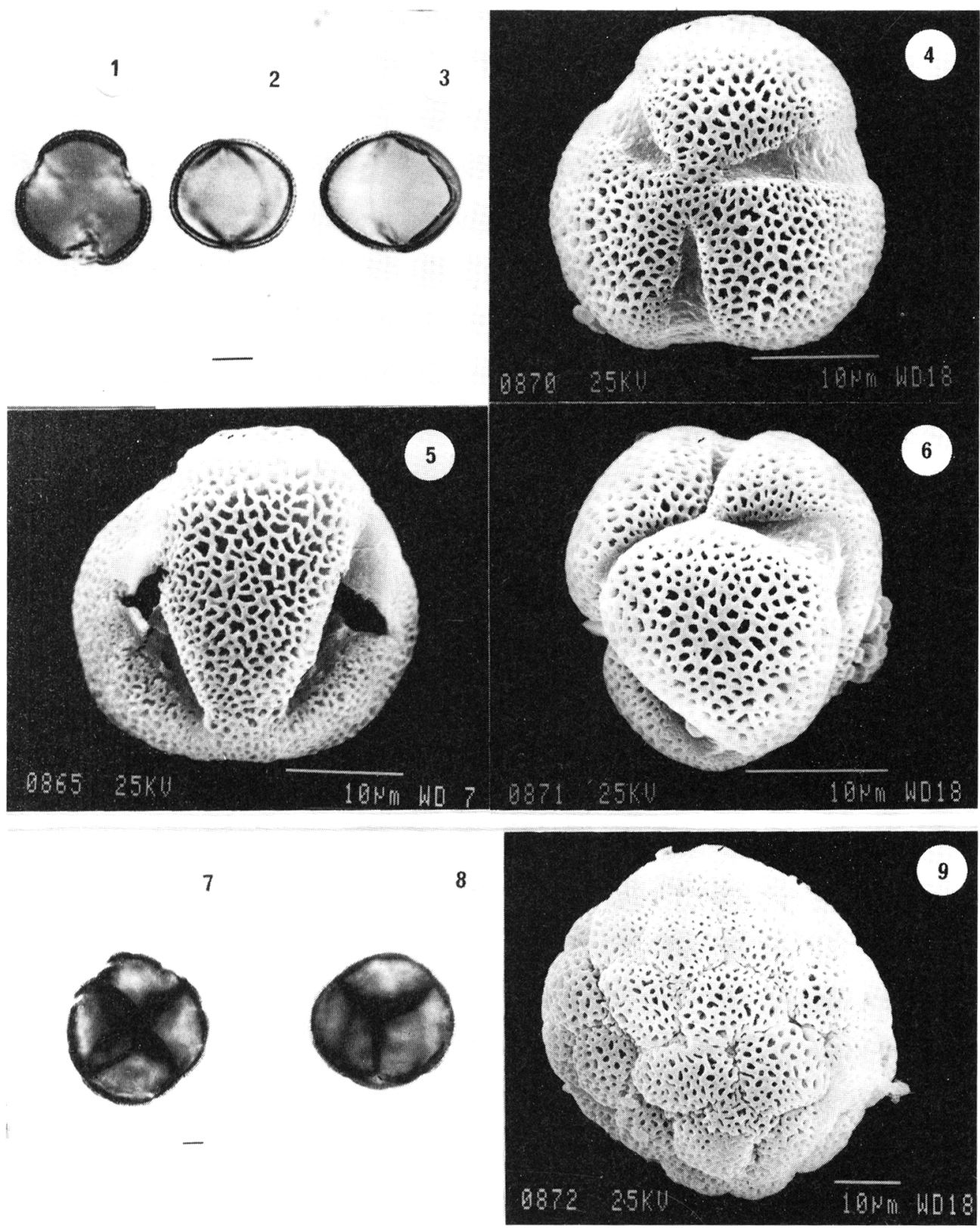

Lám. I.- Campsis radicans: 1, vista polar y corte óptico ecuatorial. 2 y 3, vista ecuatorial y corte óptico meridiano. 4, aspecto general, vista polar. Campsis grandiflora: 5, aspecto general, vista ecuatorial. Calalpa bignonioides: 7 y 8, tétrade, corte óptico. 9, tétrade, aspecto general. Escala MO: $10 \mu \mathrm{m}$. 


\section{RESULTADOS}

Campsis grandiflora Loisel (China y Japón).

Campsis radicans Seem. (sur de Estados Unidos).

Polen, generalmente, trizonocolporado, aunque es bastate frecuente la aparición de tipos tetracolpados, loxocolpados y sincolpados en uno o en ambos polos. Isopolar o, a veces, heteropolar, radiosimétrico. Circular en vista polar y corte óptico ecuatorial; de subcircular a ligeramente elíptico en vista ecuatorial y corte óptico meridiano, de suboblado a proladoesferoidal, siendo $\mathrm{P} / \mathrm{E}=0,90-1,14(1,02 \pm 0,08)$ en $C$. gradiflora y $0,81-1,07(0,90 \pm 0,05)$ en C. radicans. Tamaño de pequeño a mediano, con valores de $\mathrm{P}=22-29(25,63 \pm 1,70) \mu \mathrm{m}$ en C. grandiflora y $21-25,5(23,13 \pm 1,03) \mu \mathrm{m}$ en $C$. radicans y de $\mathrm{E}=21,5-29(25,27 \pm 2,19) \mu \mathrm{m}$ en $C$. grandiflora y $22-28(25,66 \pm 1,30) \mu \mathrm{m}$ en $C$. radicans. Aberturas compuestas; ectoaberturas de tipo colpo, terminales, con la membrana apertural psilada; endoaberturas de tipo poro, lalongadas. Exina de 1,2 a 1,6 $\mu \mathrm{m}$ de grosor, con la sexina de 1,5 a 2 veces más gruesa que la nexina, engrosándose ésta última ligeramente a nivel de las endoaberturas para formar costillas. Téctum parcial. Infratéctum columelado, con columelas gruesas y ensanchadas en la parte apical. Superficie reticulada, con lúmenes irregulares de hasta $1,5 \mu \mathrm{m}$ de longitud, decreciendo el tamaño de los lúmenes a medida que se aproximan a los colpos; muros psilados. (Lám. I, figs. 1-6).

Referencias: se desconocen.

Catalpa bignonioides Walt. (este de Estados Unidos).

Polen polipantosincolpado, agrupado en tétradas tetraédricas regulares de 55-72 $(61,02 \pm 4,19) \mu \mathrm{m}$ de diámetro, siendo las monadas de tamaño mediano con valores de $\mathrm{D}=33$ $50(43,55 \pm 4,66) \mu \mathrm{m}$. Aberturas simples, de tipo colpo, de 8 a $12 \mu \mathrm{m}$ de longitud y fusionadas de 3 en 3; membrana apertural difícil de apreciar debido a la escasa amplitud que presentan los colpos. Exina de 3 - 3,5 $\mu \mathrm{m}$ de grosor, con la sexina de 3 a 4 veces más gruesa que la nexina. Téctum parcial. Infratéctum columelado, con las columelas gruesas. Superficie reticulada, con lúmenes irregulares de hasta $1 \mu \mathrm{m}$ de grosor; muros psilados. (Lám. I, figs. 7-9).

Referencias: Erdtman (1952), Lewis et al. (1983), Díaz de la Guardia et al. (1990)

\section{Doxantha unguis-cati Rehd. (Argentina y Brasil).}

Polen, generalmente, trizonocolporado (colporoidado), aunque es bastante frecuente la aparición de tipos tetracolpados, loxocolpados, sincolpados en uno o en los dos polos, e incluso con los colpos ecuatoriales unidos en una o en ambas zonas polares a tres sincolpos. Isopolar o, a veces, heteropolar, radiosimétrico. Circular en vista polar y corte óptico ecuatorial; de circular a ligeramente elíptico en vista ecuatorial y corte óptico meridiano, de oblado a prolado-esferoidal, siendo $\mathrm{P} / \mathrm{E}=0,74-1,05(0,87 \pm 0,07)$. Tamaño mediano, con valores de $\mathrm{P}=25-39(30,64 \pm 2,41) \mu \mathrm{m}$ y de $\mathrm{E}=29-44(35,01 \pm 2,80) \mu \mathrm{m}$. Aberturas compuestas; ectoaberturas de tipo colpo, terminales, con la membrana apertural psilada; endoaberturas de tipo poro, bastante difusas. Exina de, aproximadamente, $2 \mu \mathrm{m}$ de grosor, con la sexina de 2 a 2,5 veces más gruesa que la nexina. Téctum casi completo. Infratéctum columelado. Superficie perforado-reticulada. (Lám. II, figs. 1-7).

Referencias: se desconocen.

Jacaranda acutifolia Humb. \& Bompl. (Brasil).

Polen trizonocolpado. Isopolar, radiosimétrico. Circular lobulado en vista polar y 

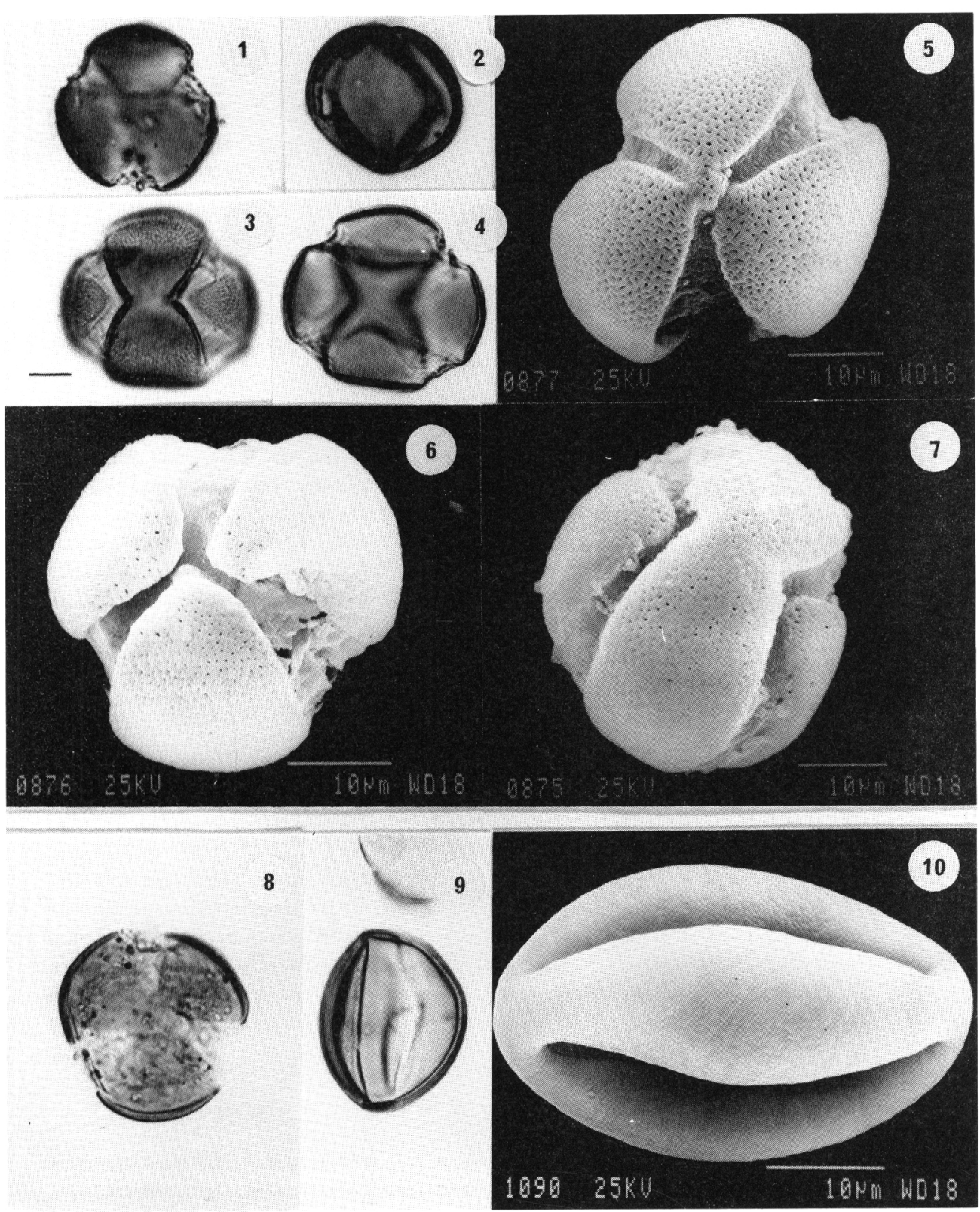

Lám. II.- Doxantha unguis-cati: 1, vista polar y corte óptico ecuatorial. 2, vista ecuatorial y corte óptico meridiano. 3, vista polar, superficie. 4 , vista polar y corte ópticoecuatorial. 5 y 6 , aspecto general, vista polar. 7, aspecto general, vista ecuatorial.Jacaranda acutifolia: 8, vista polar y corte óptico ecuatorial.9, vista ecuatorial y corte óptico meridiano. 10, aspecto general, vista ecuatorial. Escala MO: $10 \mu \mathrm{m}$ 
corte óptico ecuatorial; elíptico en vista ecuatorial y corte óptico meridiano, de obladoesferoidal a prolado, siendo $\mathrm{P} / \mathrm{E}=0,91-1,61(1,20 \pm 1,19)$. Tamaño mediano, con valores de $\mathrm{P}=33-41(36,41 \pm 2,54) \mu \mathrm{m}$ y de $\mathrm{E}=24-38(30,89 \pm 5,16) \mu \mathrm{m}$. Aberturas simples, de tipo colpo, terminales, con la membrana apertural psilado-perforada. Exina de 2 a 2,5 $\mu \mathrm{m}$ de grosor, con la sexina 2 veces más gruesa que la nexina. Téctum completo. Infratéctum columelado, con las columelas poco patentes. Superficie psilado-perforada. (Lám. II, figs. 8-10).

Referencias: Gálvez y Ubera (1985), Mateu et al. (1987).

Pandorea jasminoides Schum. (Australia).

Pandorea pandorana Van Steenis (Australia y Malasia).

Polen trizonocolporado (colporoidado). Isopolar, radiosimétrico. Circular lobulado en vista polar y corte óptico ecuatorial; de circular a ligeramente elíptico en vista ecuatorial y corte óptico meridiano, de suboblado a subprolado, siendo $\mathrm{P} / \mathrm{E}=0,83-1,09(0,96 \pm 0,08)$ en $P$. jasminoides y $0,88-1,15(1,01 \pm 0,07)$ en $P$. pandorana. Tamaño mediano, con valores de $\mathrm{P}=$ 25-31 (27,55 $\pm 2,02) \mu \mathrm{m}$ en $P$. jasminoides y $31-38(34,6 \pm 2,10) \mu \mathrm{m}$ en $P$. pandorana y de $\mathrm{E}=$ 25-30 $(28,57 \pm 1,43) \mu \mathrm{m}$ en $P$. jasminoides y $31-38(34,2 \pm 1,64) \mu \mathrm{m}$ en $P$. pandorana. Aberturas compuestas; ectoaberturas de tipo colpo, terminales, con la membrana apertural psilada; endoaberturas de tipo poro, bastante difusas. Exina de 2,5 a $3 \mu \mathrm{m}$ de grosor en $P$. jasminoides y de aproximadamente $2 \mu \mathrm{m}$ en $P$. pandorana, con la sexina de 2 a 3 veces más gruesa que la nexina. Téctum parcial. Infratéctum columelado, con columelas gruesas y ensanchadas en la parte apical. Superficie reticulada, con lúmenes irregulares de hasta $2 \mu \mathrm{m}$ de longitud, reduciéndose en tamaño a medida que se aproximan a los colpos en cuyas inmediaciones la superficie se hace perforada; muros psilados. (Lám. III, figs. 1-4).

Referencias: se desconocen.

\section{Podranea ricasoliana Sprange (Sudáfrica).}

Polen trizonocolporado (colporoidado). Isopolar, radiosimétrico. Circular lobulado en vista polar y corte óptico ecuatorial; de circular a elíptico en vista ecuatorial y corte óptico meridiano, de oblado a prolado-esferoidal, siendo $\mathrm{P} / \mathrm{E}=0,64-1,11(0,82 \pm 0,12)$. Tamaño mediano, con valores de $\mathrm{P}=27-39(32,48 \pm 3,57) \mu \mathrm{m}$ y de $\mathrm{E}=35-45(39,92 \pm 2,34) \mu \mathrm{m}$. Aberturas compuestas; ectoaberturas de tipo colpo, terminales, con la membrana apertural psilada; endoaberturas de tipo poro, bastante difusas. Exina de aproximadamente $3 \mu \mathrm{m}$ de grosor, con la sexina de 2 a 3 veces más gruesa que la nexina. Téctum parcial. Infratéctum columelado, con columelas gruesas y ensanchadas en la parte apical. Superficie reticulada, con lúmenes irregulares de hasta $3,5 \mu \mathrm{m}$ de longitud, decreciendo en tamaño a medida que se aproximan a los colpos; muros ondulados, pudiéndose apreciar algunas columelas libres en la parte central de los lúmenes. (Lám. III, figs. 5-9).

Referencias: se desconocen.

\section{Pyrostegia ignea Presl. (Brasil).}

Polen, generalmente, 12-pantocolpado, con 4 colpos ecuatoriales unidos a 4 sincolpos en ambos polos $(4,4,4)$, habiéndose encontrado con bastante frecuencia tipos tetrazonocolpados y trizonocolpados unidos en uno o en ambos polos a 3 sincolpos. Generalmente isopolar, a veces heteropolar debido al número y disposición de los colpos polares, radiosimétrico. Circular en vista polar y corte óptico ecuatorial; de subcircular a ligeramente elíptico en vista ecuatorial y corte óptico meridiano, de prolado-esferoidal a prolado, siendo $\mathrm{P} / \mathrm{E}=1-1,35(1,19$ $\pm 0,09)$. Tamaño grande, con valores de $\mathrm{P}=60-72(64,07 \pm 2,99) \mu \mathrm{m}$ y de $\mathrm{E}=48-65(54,14 \pm$ 

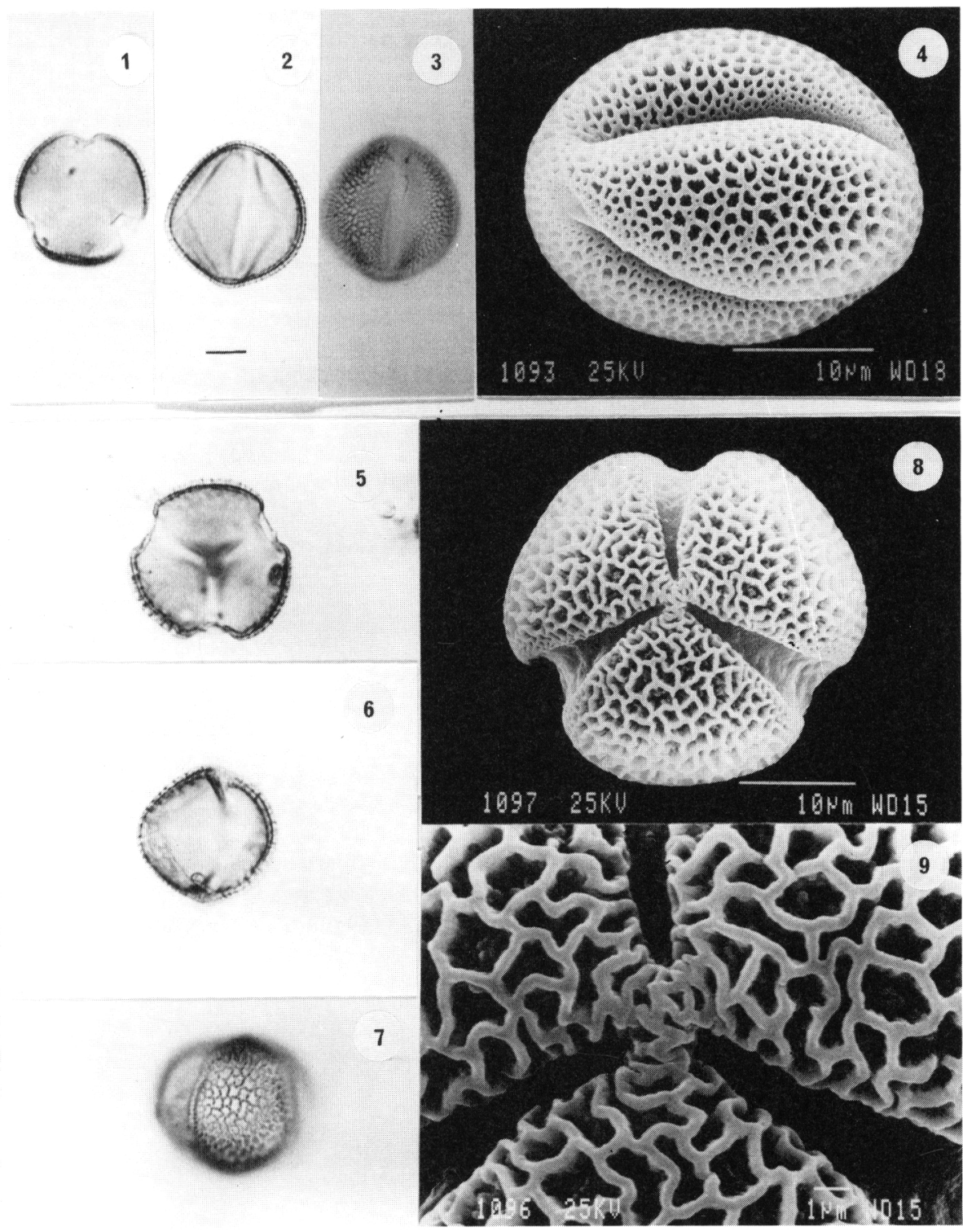

Lám. III.- Pandorea pandorana: 1, vista polar y corte óptico ecuatorial. 2, vista ecuatorial y corte óptico meridiano. 3, vista ecuatorial, abertura y superficie. Pandorea jasminoides: 4, aspecto general. Podranea ricasoliana: 5, vista polar y corte óptico ecuatorial. 6, vista ecuatorial y corte óptico meridiano. 7, superficie. 8, aspecto general, vista polar. 9, detalle de la superficie. Escala MO: $10 \mu \mathrm{m}$. 
$4,81) \mu \mathrm{m}$. Exina de 3 a $4 \mu \mathrm{m}$ de grosor, con la sexina 2 veces más gruesa que la nexina. Téctum completo. Infratéctum columelado. Superficie verrucosa, con verrugas irregulares y huecas que, al fracturarse, dan lugar a lúmenes irregulares que no llegan a formar retículo. (Lám. IV, figs. 1-5).

Referencias: se desconocen.

Spathodea campanulata Beauv. (Africa ecuatorial).

Polen trizonocolporado. Isopolar, radiosimétrico. Circular lobulado en vista polar y corte óptico ecuatorial; de subcircular a elíptico en vista ecuatorial y corte óptico meridiano, de oblado-esferoidal a subprolado siendo $\mathrm{P} / \mathrm{E}=0,95-1,30(1,14 \pm 0,09)$. Tamaño mediano, con valores de $\mathrm{P}=39-54(47,62 \pm 3,46) \mu \mathrm{m}$ y de $\mathrm{E}=38-44(41,83 \pm 2,03) \mu \mathrm{m}$. Aberturas compuestas; ectoaberturas de tipo colpo, terminales, con la membrana apertural psilada; endoaberturas de tipo poro, lalongadas. Exina de 2,5 a $3 \mu \mathrm{m}$ de grosor, con la sexina 1,5 veces más gruesa que la nexina. Téctum parcial. Infratéctum columelado, con columelas gruesas. Superficie reticulada, con lúmenes irregulares de hasta 1,5 $\mu \mathrm{m}$ de longitud; muros psilados. (Lám. IV, figs. 6-9).

Referencias: Huang (1970).

Tecoma stans H.B.K. (América Central y del Sur).

Polen trizonocolpado, a veces trizonosincolpado, agrupado en tétrades tetraédricas regulares de 56-72 $(66,5 \pm 6,37) \mu \mathrm{m}$ de diámetro, siendo las monadas de tamaño mediano con valores de $\mathrm{D}=24-36(31 \pm 5,51) \mu \mathrm{m}$. Aberturas simples, de tipo colpo, con la membrana apertural psilada. Exina de $1 \mu \mathrm{m}$ o menos de grosor, con la sexina 2 veces más gruesa que la nexina. Téctum completo. Infratéctum columelado, con las columelas poco patentes. Superficie psilado-perforada, a veces escábrida. (Lám. V, figs. 1 y 2).

Referencias: se desconocen.

Tecomaria capensis Spach. (Sudáfrica).

Polen trizonocolporado (colporoidado). Isopolar, radiosimétrico. De circular a subtriangular en vista polar y corte óptico ecuatorial; de circular a ligeramente elíptico en vista ecuatorial y corte óptico meridiano, de oblado-esferoidal a subprolado siendo $\mathrm{P} / \mathrm{E}=0,88-1,25$ $(0,99 \pm 0,07)$. Tamaño mediano, con valores de $\mathrm{P}=36-45(39,68 \pm 2,37) \mu \mathrm{m}$ y de $\mathrm{E}=38-43(40$, $08 \pm 1,46) \mu \mathrm{m}$. Aberturas compuestas; ectoaberturas de tipo colpo, terminales, con la membrana apertural rugosa; endoaberturas de tipo poro, bastante difusas. Exina de $2-2,5 \mu \mathrm{m}$ de grosor, con la sexina de 1,5 a 2 veces más gruesa que la nexina. Téctum parcial. Infratéctum columelado. Superficie reticulada, con lúmenes inferiores a $1 \mu \mathrm{m}$ de diámetro; muros psilados. (Lám. V, figs. 3-7).

Referencias: se desconocen.

\section{DISCUSIÓN}

En las especies estudiadas, el tipo polínico que más frecuentemente aparece es el trizocolporado y el trizonocolporoidado, si bien se pueden encontrar, incluso dentro de la misma especie, tipos tetracolpados, loxocolpados, sincolpados en uno o en ambos polos e incluso de granos de polen con 3 ó 4 colpos ecuatoriales unidos en uno o en ambos polos a 3 ó 4 sincolpos respectivamente $(3,3,3 ; 4,4,4)$. Así ocurre en 


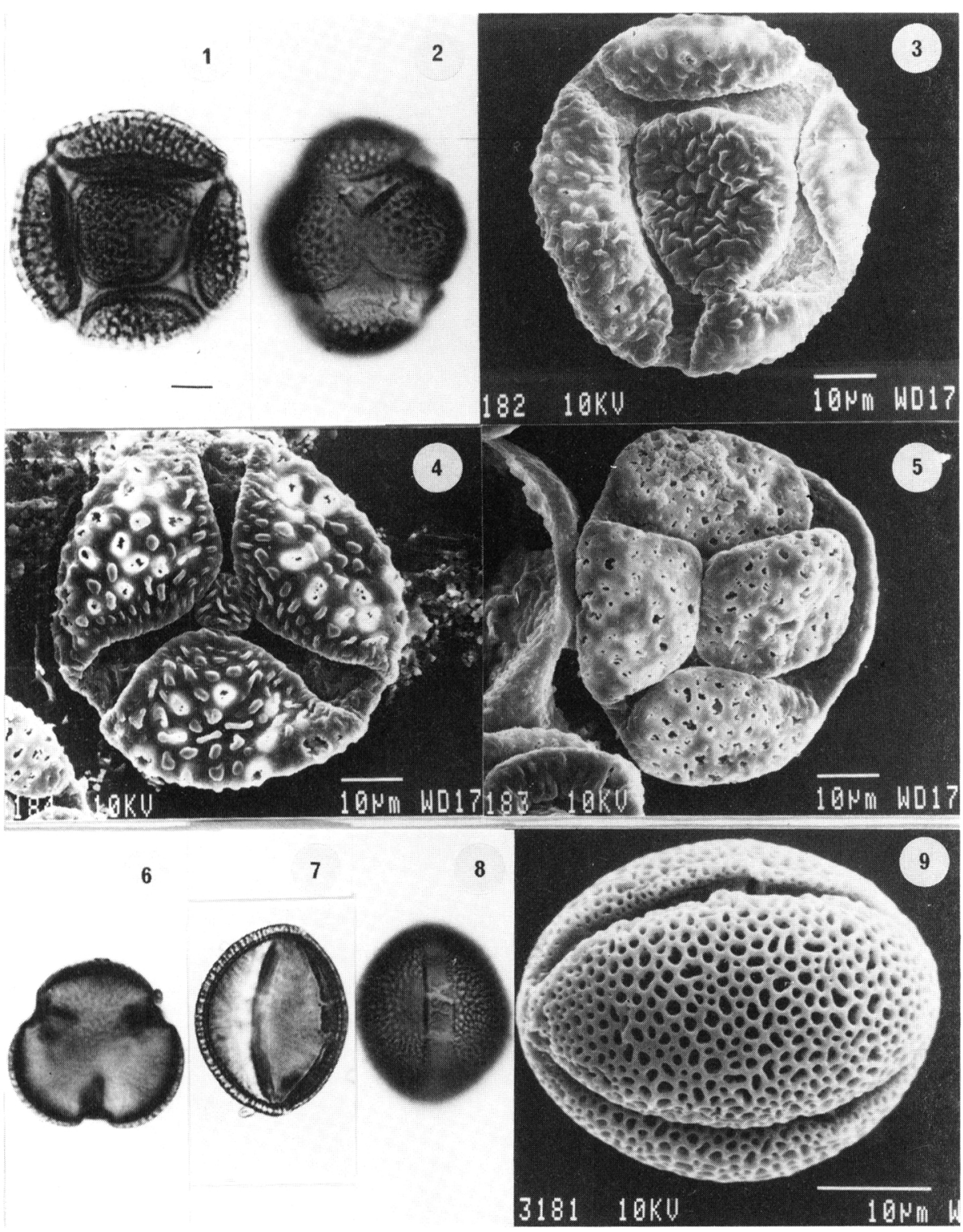

Lám. IV.- Pyrostegia ignea: 1, vista polar y corte óptico ecuatorial. 2, vista polar, aberturas y superficie. 3, y 4, aspecto general, vista polar. 5, aspecto general, vista ecuatorial. Spathodea campanulata: 6, vista polar y corte óptico ecuatorial. 7, vista ecuatorial y corte óptico meridiano. 8 , vista ecuatorial, abertura y superficie. 9, aspecto general, vista ecuatorial. Escala MO: $10 \mu \mathrm{m}$. 
Campsis radicans, Campsis grandiflora, Doxantha unguis-cati y Pyrostegia ignea. Por otra parte, Catalpa bignoniodes y Tecoma stans presentan el polen unido en tétrades.

El tamaño oscila de mediano a grande, y las aberturas son colpadas, colporadas o colporoidadas, si bien hemos podido apreciar que las endoaberturas, a veces bastante difusas, se sitúan, más que en la parte central del colpo, desplazadas a ambos lados de esta zona. Esto último está en relación con discontinuidades observadas en la nexina cuando se aprecian los granos de polen en vista ecuatorial y corte óptico meridiano (Lám. I, figs. 3 y 4; Lám. IV, fig. 7)). El carácter colporoidado de las aberturas ya fue puesto anteriormente de manifiesto en especies de esta familia por Ferguson \& Santisuk (1973).

En cuanto a la estratificación de la exina, la sexina siempre aparece más gruesa que la nexina y la superficie suele ser reticulada o perforado-reticulada, salvo en Jacaranda acutifolia y en Tecoma stans, en que es psilado-perforada.

A pesar de ser escasas las referencias que tenemos sobre la morfología polínica de las especies aquí estudiadas, se han encontrado algunas diferencias con la bibliografía consultada en cuanto a la interpretación del polen se refiere. Así, Lewis et al. (1983), describen el polen de Catalpa como inaperturado y aerolado y Díaz de la Guardia et al. (1990) como trizonosincolpado, estando nuestras observaciones más de acuerdo con Erdtman (1952) en que se trata de una tétrade. Por otra parte, Galvez y Ubera (1985), describieron las aberturas de Jacaranda acutifolia compuestas de colpo y poro, cuando parece ser que son simples y colpadas. Por último, el polen de Spathodea campanulata fue considerado por Huang (1970) como colpado mientras que a nosotros nos aparece colporado, si bien las endoaberturas no se encuentran exactamente situadas en la parte central del colpo, hecho que también hemos podido apreciar en otras especies (Campsis grandiflora, C.radicans); este mismo autor encontró que la nexina del polen de esta especie era más gruesa que la sexina cuando en las muestras por nosotros estudiadas ocurre justamente lo contrario.

Aunque no se pueden hacer consideraciones taxonómicas habiéndose estudiado tan pocas especies, parece serque la morfología polínica es un buen caracter a la hora de diferencias géneros dentro de la familia Bignoniaceae habida cuenta de que aquellas especies incluidas dentro de un mismo género poseen un tipo de polen similar y que especies de géneros diferentes son perfectamente diferenciables en base al tipo polínico que presentan.

Material examinado. Campsis grandiflora: Málaga, Carretera Málaga-Coín. 22-6-85 (MGC 16966). Campsis radicans: Málaga, Paseo de Martiricos. 26-6-85 (MGC 16976). Catalpa bignonioides: Málaga, Avda. de Andalucía. 2-6-85 (16965). Doxantha unguis-cati: Málaga, Urbanización El Limonar. 12-5-85 (MGC 16968). Jacaranda acutifolia: Málaga, Urbanización Las Palmeras. 26-5-85 (MGC 16970). Málaga, Urbanización El Limonar. 26-5-85 (MGC 16971). Pandorea jasminoides: Málaga, Urbanización El Limonar. 26-5-85 (MGC 16972). Pandorea pandorana: Málaga, Urbanización El Limonar. 28-2-85 (MGC16975). Podranea ricasoliana: Málaga, Urbanización El Limonar. 28-1-85 (MGC 16976). Málaga, antigua 

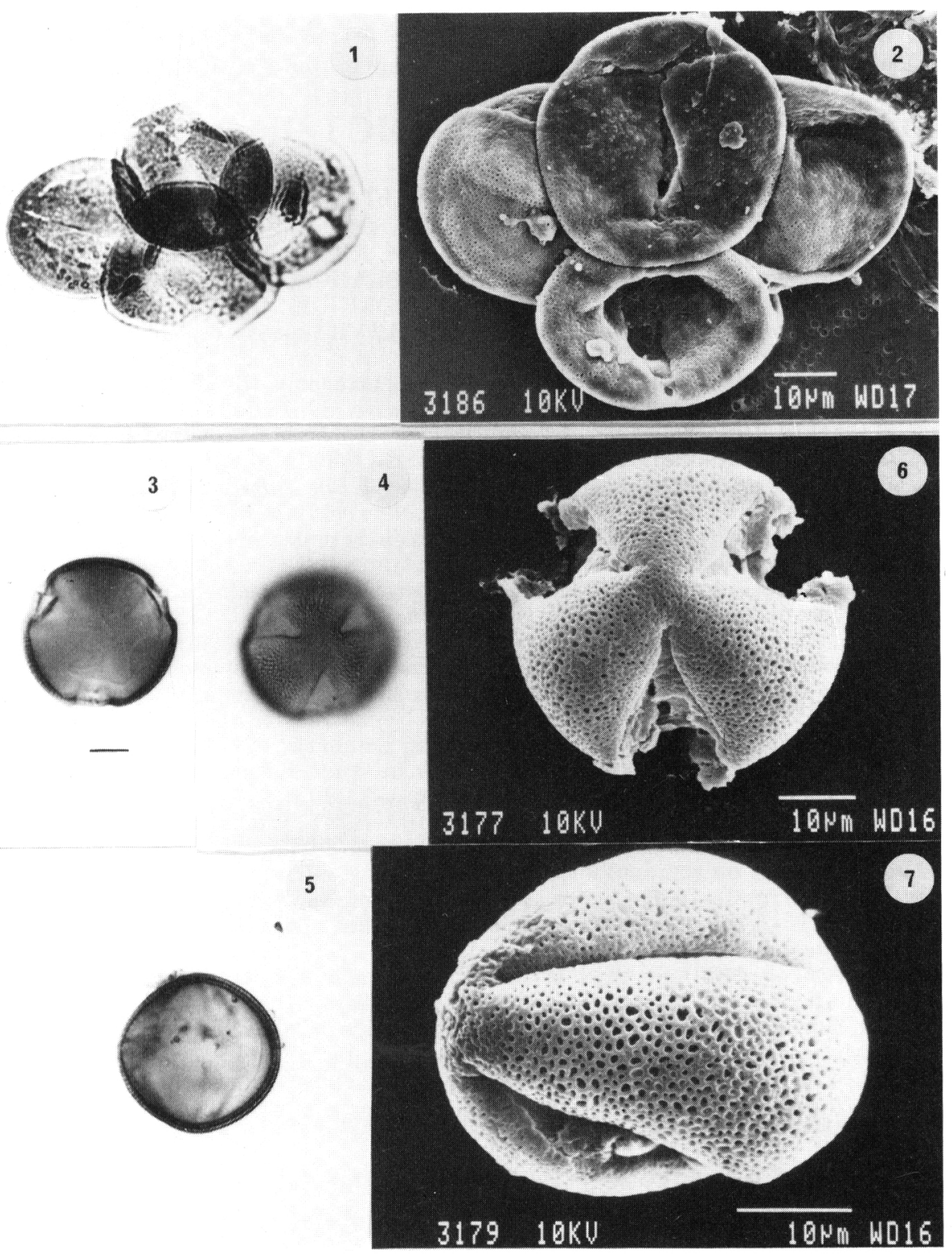

Lam.V.-Tecoma stans: 1,tétrade, corteóptico. 2, aspecto general de la tétrade.Tecomaria capensis: 3 , vista polar y corte óptico ecuatorial. 4,vista polar, superficie. 5, vista ecuatorial y corte óptico meridiano. 6, aspecto general, vista polar. 7, aspecto general, vista ecuatorial. Escala MO: $10 \mu \mathrm{m}$. 
Facultad de Ciencias. 27-1-85 (MGC 16977). Pyrostegia ignea: Málaga, Valleniza, jardines del Vivero A-1. 27-2-86 (MGC 16641). Spathodea campanulata: Málaga, El Parque. 9-9-85 (MGC 16978). Tecoma stans: Málaga, Paseo de Reding. 16-6-85 (MGC 17241). Tecomaria capensis: Málaga, Urbanización El Limonar. 28-2-85 (MGC 16981).

\section{BIBLIOGRAFÍA}

DIAZ DE LA GUARDIA, C., G. BLANCA y R.M. NIETO -1990- Palinología en plantas ornamentales de la ciudad de Granada. En G. BLANCA, C. DIAZ DE LA GUARDIA, M.C. FERNÁNDEZ, M. GARRIDO, M. I. RODRÍGUEZ-GARCÍA y A. T. ROMERO (eds.). Polen, esporas y sus aplicaciones. Actas del VII Simposio de Palinología: 347-354. Servicio de Reprografía de la Facultad de Ciencias, Granada.

ERDTMAN, G. -1945- Polen morphology and plant taxonomy. III. Morina L. with addition on morphological terminology. Svensk. Bot. Tidskr., 39: 187-191.

ERDTMAN, G. -1952-Pollen morphology and plant taxonomy. Angiosperms (An introducction to palynology). Hafner Publ. Co., Stockholm.

ERDTMAN, G. -1960- The acetolysis method. Svensk. Bot. Tidskr., 54:561-564.

FAEGRI, K. \& J. IVERSEN -1975- Texbook of pollen analysis. Munksgaard, Copenhagen.

FERGUSON, I.K. \& T. SANTISUK -1973- Notes on the pollen morphology of some Asiatic Bignoniaceae. Kew Bulletin 28(2):187-198.

GÁLVEZ C. y J.L. UBERA -1985- Nuevas aportaciones al catálogo aeropalinológico de Córdoba: plantas exóticas. Anales Asoc. Palinol. Lengua Esp. 2:215-225.

HIDEUX, M. \& I.K. FERGUSON -1975- Stereostructure de l'exine des Saxifragales: proposition d'une description logique et schematique. Bull. Soc. Bot. France, 122:57-67.

HUANG, T.C. -1970- Pollen grains of formosan plants (6). Taiwania, 15:13-179.

LEWIS, W.H., P. VINAY \& V.E. ZENGER -1983-Airborne and allergenic pollen of North America. The Johns Hopkins University Press, Baltimore.

MATEU, I., A. AGUILELLA y J.M. AZCARRAGA -1987- Pólenes del Jardín Botánico de Valencia. I. Anales Asoc. Palinol. Lengua Esp. 3:73-90.

SUAREZ-CERVERA, M. y J.A. SEOANE-CAMBA -1983-. Estudio del contenido polínico de la atmósfera de Barcelona según un nuevo método de filtración. Collectaena Botanica 14:587-615.

SWINEFORD Jr., C. -1940- Catalpa as a cause of hay fever. J. Allergy 11:398-401.

REITSMA, T. - 1969- Size modification of recent pollen grains under different treatments. Rev. Paeobot. Palynol., 9:175-202.

REITSMA, T. - 1970- Suggestions towards unification of descriptive terminology of Angiosperms pollen grains. Rev. Paleobot. Palinology, 10:39-60.

TRIGO, M.M. -1989- Aportación al estudio palinológico de la flora ornamental de la ciudad de Málaga: Gimnospermas. Acta Bot. Malacitana, 14:238-244.

TRIGO, M.M. y I. GARCIA -1990- Morfología polínica de plantas ornamentales: Leguminosas. Acta Bot. Malacitana, 15:45-67.

VAN CAMPO, M. -1958-Palinologie africaine II. Bull. Inst. Franç. Afrique Noire, sér.A, Sci. Nat., 20: 753-760.

WODEHOUSE, R.P. -1935- Pollen grains. McGraw-Hill, New York. U.S.A

(Aceptado para su publicación en junio de 1991)

Dirección de la autora: Departamento de Biología Vegetal. Facultad de Ciencias. Universidad de Málaga. 29080 Málaga. 